University of Louisville

ThinkIR: The University of Louisville's Institutional Repository

Faculty Scholarship

$4-2012$

\title{
The S4G perspective on circumstellar dust extinction of asymptotic giant branch stars in M100.
}

\author{
Sharon Meidt \\ Max-Planck-Institut für Astronomie \\ Eva Schinnerer \\ Max-Planck-Institut für Astronomie \\ Juan Carlos Munoz-Mateos \\ National Radio Astronomy Observatory \\ Benne W. Holwerda \\ University of Louisville \\ Luis C. Ho \\ Observatories of the Carnegie Institution for Science \\ See next page for additional authors
}

Follow this and additional works at: https://ir.library.louisville.edu/faculty

Part of the Astrophysics and Astronomy Commons

Original Publication Information

Meidt, Sharon E., et al. "The S4G Perspective on Circumstellar Dust Extinction of Asymptotic Giant Branch Stars in M100." 2012. The Astrophysical Journal Letters 748(2): 6 pp.

This Article is brought to you for free and open access by ThinkIR: The University of Louisville's Institutional Repository. It has been accepted for inclusion in Faculty Scholarship by an authorized administrator of ThinkIR: The University of Louisville's Institutional Repository. For more information, please contact thinkir@louisville.edu. 


\section{Authors}

Sharon Meidt, Eva Schinnerer, Juan Carlos Munoz-Mateos, Benne W. Holwerda, Luis C. Ho, Barry F. Madore, Johan H. Knapen, Albert Bosma, E. Athanassoula, Joannah L. Hinz, Kartik Sheth, Michael Regan, Armando Gil de Paz, Karin Menendez-Delmestre, Mark Seibert, Taehyun Kim, Trisha Mizusawa, Dimitri A. Gadotti, Eija Laurikainen, Heikki Salo, Jarkko Laine, and Sebastien Comeron 


\title{
THE $S^{4} G$ PERSPECTIVE ON CIRCUMSTELLAR DUST EXTINCTION OF ASYMPTOTIC GIANT BRANCH STARS IN M100
}

\author{
Sharon E. Meidt ${ }^{1}$, Eva Schinnerer ${ }^{1}$, Juan-Carlos Muñoz-Mateos ${ }^{2}$, Benne Holwerda ${ }^{3}$, Luis C. Ho ${ }^{4}$, Barry F. Madore $^{4}$, \\ Johan H. Knapen 5,6 , Albert Bosma ${ }^{7}$, E. Athanassoula ${ }^{7}$, Joannah L. Hinz ${ }^{8}$, Kartik Sheth $^{4,9,10}$, Michael Regan ${ }^{11}$, \\ Armando Gil de Paz ${ }^{12}$, Karín Menéndez-Delmestre ${ }^{4}$, Mark Seibert ${ }^{4}$, Taehyun Kim² ${ }^{2}$, Trisha Mizusawa ${ }^{9,10}$, \\ Dimitri A. Gadotti ${ }^{13}$, Eija Laurikainen ${ }^{14,15}$, Heikki Salo $^{14}$, JarkKo Laine ${ }^{14,15}$, and Sébastien ComeróN ${ }^{16}$ \\ ${ }^{1}$ Max-Planck-Institut für Astronomie/Königstuhl 17, D-69117 Heidelberg, Germany \\ ${ }^{2}$ National Radio Astronomy Observatory, Charlottesville, VA, USA \\ ${ }^{3}$ European Space Agency, ESTEC, Keplerlaan 1, 2200 AG, Noordwijk, the Netherlands \\ ${ }^{4}$ The Observatories of the Carnegie Institution for Science, Pasadena, CA, USA \\ ${ }^{5}$ Instituto de Astrofisica de Canarias, Tenerife, Spain \\ ${ }^{6}$ Departamento de Astrofísica, Universidad de La Laguna, La Laguna, Spain \\ ${ }^{7}$ Laboratoire d'Astrophysique de Marseille (LAM), Marseille, France \\ ${ }^{8}$ Department of Astronomy, University of Arizona, Tucson, AZ, USA \\ ${ }^{9}$ Spitzer Science Center, Pasadena, CA, USA \\ ${ }^{10}$ California Institute of Technology, Pasadena, CA, USA \\ ${ }^{11}$ Space Telescope Science Institute, Baltimore, MD, USA \\ ${ }^{12}$ Departamento de Astrofísica, Universidad Complutense Madrid, Madrid, Spain \\ ${ }^{13}$ European Southern Observatory, Santiago, Chile \\ ${ }^{14}$ Astronomy Division, Department of Physical Sciences, University of Oulu, Oulu, Finland \\ ${ }^{15}$ Finnish Centre for Astronomy with ESO (FINCA), University of Turku, Piikkiö, Finland \\ ${ }^{16}$ Korea Astronomy and Space Science Institute, Daejeon, Republic of Korea \\ Received 2011 December 18; accepted 2012 February 29; published 2012 March 19
}

\begin{abstract}
We examine the effect of circumstellar dust extinction on the near-IR (NIR) contribution of asymptotic giant branch (AGB) stars in intermediate-age clusters throughout the disk of M100. For our sample of 17 AGB-dominated clusters we extract optical-to-mid-IR spectral energy distributions (SEDs) and find that NIR brightness is coupled to the mid-IR dust emission in such a way that a significant reduction of AGB light, of up to 1 mag in the $K$ band, follows from extinction by the dust shell formed during this stage. Since the dust optical depth varies with AGB chemistry (C-rich or O-rich), our results suggest that the contribution of AGB stars to the flux from their host clusters will be closely linked to the metallicity and the progenitor mass of the AGB star, to which dust chemistry and mass-loss rate are sensitive. Our sample of clusters — each the analogue of a $\sim 1$ Gyr old post-starburst galaxy-has implications within the context of mass and age estimation via SED modeling at high- $z$ : we find that the average $\sim 0.5$ mag extinction estimated here may be sufficient to reduce the AGB contribution in the (rest-frame) $K$ band from $\sim 70 \%$, as predicted in the latest generation of synthesis models, to $\sim 35 \%$. Our technique for selecting AGB-dominated clusters in nearby galaxies promises to be effective for discriminating the uncertainties associated with AGB stars in intermediate-age populations that plague age and mass estimation in high- $z$ galaxies.
\end{abstract}

Key words: galaxies: individual (M100) - galaxies: star clusters: general - galaxies: stellar content - stars: AGB and post-AGB

Online-only material: color figures

\section{INTRODUCTION}

Light emitted from stars in the thermally pulsing asymptotic giant branch (AGB) phase of stellar evolution has implications for our view of the baryonic content and chemical state of galaxies, both locally and at high redshift, from UV-to-midIR observations. Recent advances in modeling and observation have enhanced this perspective, especially regarding the dust formation and mass loss that accompanies the late (thermal pulsing) stages of AGB evolution (e.g., Groenewegen 2006; Jackson et al. 2007; Groenewegen et al. 2009; Boyer et al. 2011). These findings demonstrate the influential role AGBs play in the enrichment of the interstellar medium (ISM) with gas and dust (e.g., Srinivasan et al. 2009; Matsuura et al. 2009; Sargent et al. 2010) and place more accurate constraints on AGB lifetimes (i.e., Girardi \& Marigo 2007).

However, our view of stellar populations as a whole has only slowly incorporated this picture, and uncertainties in the ages and masses of intermediate-age systems remain. Because they are some of the brightest objects between ages $0.2-1 \mathrm{Gyr}$, a handful of AGB stars can outshine all other stars at rest-frame wavelengths $\lambda \gtrsim 1 \mu \mathrm{m}$ while contributing only minimally to the total mass. Accurate AGB models are therefore a critical ingredient in stellar population synthesis (SPS) techniques (Mouhcine \& Lançon 2002; Bruzual \& Charlot 2003; Maraston 2005; Gonzalez-Lopezlira 2010). But, even the latest generation of SPS models with updated treatment of the AGB phase (e.g., Charlot \& Bruzual 2003; Marigo et al. 2008) rarely extend beyond $3 \mu \mathrm{m}$, and few include the effect of extinction by the AGB star's circumstellar dust shell on the integrated properties of the stellar population (but see Mouhcine 2002).

Here we examine whether recent evidence in favor of a reduced near-IR (NIR) AGB contribution (Kriek et al. 2010) arises from the extinction of AGBs by their dusty envelopes. With a view extending from the optical to the mid-IR, we study a sample of AGB-dominated clusters in M100 selected for, and 
classified by, their dust emission. This avoids the challenges of optical or NIR detection and classification in the presence of the dust shell (e.g., van Loon et al. 2005) and supplies unique leverage on the role of dust to shift AGB light from the NIR to longer wavelengths.

\section{THE DATA}

We construct our sample of bright clusters in the disk of M100 ( $D=15.2 \mathrm{Mpc}$; Freedman et al. 2001), chosen for its low inclination and the potential to probe a range of metallicities. Objects are selected based on their emission in IRAC 3.6 and $4.5 \mu \mathrm{m}$ images processed by the $\mathrm{S}^{4} \mathrm{G}$ team (Sheth et al. 2010; see selection technique below). In order to sample the spectral energy distribution (SED) from 0.2 to $24 \mu \mathrm{m}$, we supplement these data with archival BVRI data from SINGS (Dale et al. 2005, 2007) and SINGS IRAC 5.8 and $8 \mu \mathrm{m}$ and MIPS $24 \mu \mathrm{m}$ images (Kennicutt et al. 2003). We also include HAWK-I JHK imaging provided by P. Grosbol (e.g., Grosbol \& Dottori 2011). A map of continuum-subtracted $\mathrm{H} \alpha$ from SINGS (Kennicutt et al. 2003; Calzetti et al. 2007) provides cross-check against $\mathrm{H}$ II regions/young embedded clusters.

Our cluster sample spans a large radial range in the disk, out to $R \sim 200^{\prime \prime}$ (limited by the NIR field of view). Extrapolation of the measured gradient in the nebular emission-line chemical abundances (Moustakas et al. 2010) in M100 suggests that the ISM metallicity reaches $12+\log (\mathrm{O} / \mathrm{H}) \approx 8.1$ by $R=300^{\prime \prime}$. Assuming little chemical evolution over the last $\sim 1 \mathrm{Gyr}$, this suggests that the cluster sample spans $0.27<Z / Z_{\odot}<0.6$. In what follows, we refer to radial position and metallicity synonymously.

\subsection{Selection of Cluster Candidates}

The identification of red clusters at 3.6 and $4.5 \mu \mathrm{m}$ is based on the Independent Component Analysis (ICA) of the images at these wavelengths (see Meidt et al. 2012). ICA separates the light from the oldest stars, with colors [3.6] $-[4.5]<0$, from the emission contributed by contaminants, with colors $0<[3.6]-[4.5] \lesssim 1.5$. Candidate bright red clusters, which fall into the second category, are distinguishable from the other main sources of contaminant emission at $3.6 \mu \mathrm{m}$ given the very different relation to the non-stellar emission $F_{8, \text { ns }}$ at $8 \mu \mathrm{m}$.

Using the selection criterion $F_{3.6} / F_{8, \text { ns }}>0.3$ to avoid emission from polycyclic aromatic hydrocarbon (PAH) and hot dust as advocated by Meidt et al. (2012), we generate a map of 172 candidate clusters, shown in Figure 1. This map is used to identify the cluster emission in all wavebands, including at 3.6 and $4.5 \mu \mathrm{m}$.

\subsection{Multiband Photometry}

Photometric measurements for candidate clusters are extracted in circular apertures $6^{\prime \prime}$ in radius, sized for compatibility with the 6" PSF FWHM at $24 \mu \mathrm{m}$, our lowest angular resolution image. For this aperture size, sampling a region $444 \mathrm{pc}$ in radius at the distance of this galaxy, we adopt corrections to the infinite aperture of 1.05, 1.07, 1.08, 1.09, and 1.67 at 3.6, 4.5, 5.8, 8, and $24 \mu \mathrm{m}$, respectively, for point sources (SSC IRAC Handbook; Reach et al. 2005) and assume that these corrections are small or negligible in the optical images (i.e., Dale et al. 2007; Calzetti et al. 2007). We have confirmed that smaller apertures yield similar flux measurements for $\lambda<24 \mu \mathrm{m}$.

To isolate the cluster light from the underlying disk in each aperture we subtract local background values measured in

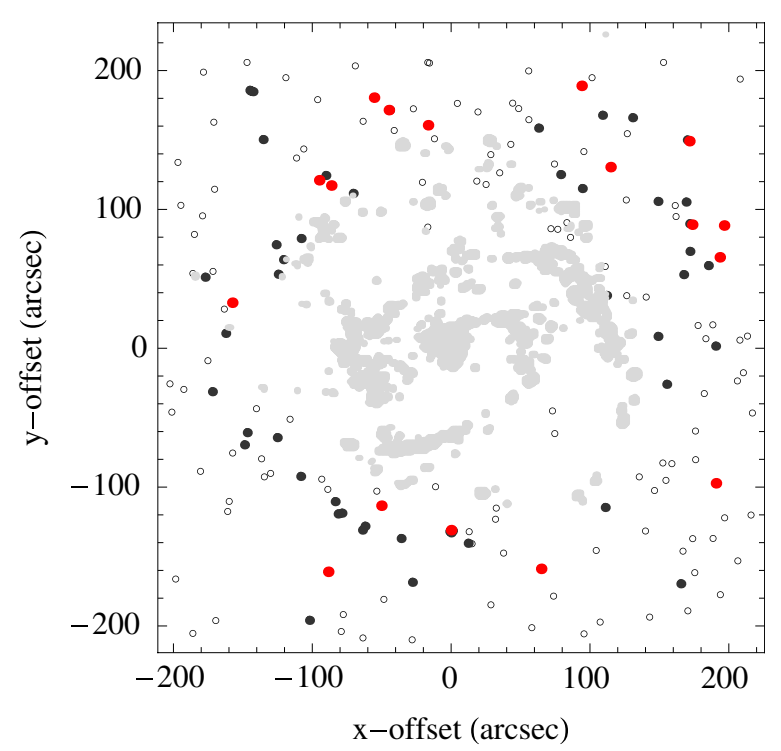

Figure 1. Spatial map of candidate clusters (open symbols), significant detections at $\lambda \geqslant 1.2 \mu \mathrm{m}$ (black), and the final sample (red), together with the hot dust/PAH emission (gray), separated as described in the text. Note that PAH and hot dust emission more often appear in the high-density, actively star-forming regions.

(A color version of this figure is available in the online journal.)

annuli spanning between $r=6^{\prime \prime}$ and $12^{\prime \prime}$ with $2 \sigma_{0}$ clipping to avoid contamination by neighboring clusters. The variance in the local background value $\sigma_{0}$ defines the uncertainty in the photometric measurement $\sigma$ for each aperture, along with calibration and aperture correction uncertainties (added in quadrature). Calibration uncertainties are $\sim 10 \%$ in the NIR bands and below this level in the IRAC bands, at MIPS $24 \mu \mathrm{m}$ and in the optical (Dale et al. 2005), while aperture corrections are typically $10 \%$ uncertain in IRAC bands and $<5 \%$ uncertain at $24 \mu \mathrm{m}$.

\section{PHOTOMETRIC PROPERTIES}

\subsection{Sample Definition}

Roughly half of the candidate clusters are detected in all bands longward of $1 \mu \mathrm{m}$. Obscuration by dust presumably contributes to some non-detections in the NIR. To ensure a realistic signal we adopt thresholding at $3 \sigma$. Measurement uncertainties for the remaining 80 significant detections are well below $20 \%$ (nearer $10 \%$ on average) and are omitted for the final sample in all upcoming plots for clarity.

Candidate clusters have some of the reddest colors in the shortest IRAC wavebands $(0<[3.6]-[4.5]<1$; Meidt et al. 2012) consistent with the colors of AGB stars. Still, possible contaminants include background galaxies, quasars, and younger dust enshrouded clusters. Galaxy number counts over the range in $3.6 \mu \mathrm{m}$ magnitudes in our sample are high (e.g., Fazio et al. 2004; Sanders et al. 2007), but only a subset has [3.6] - [4.5] colors that can be confused with emission from dusty AGBs, namely quasars and the nearest and brightest background galaxies, for which the observed mid-IR colors are closest to rest-frame colors (e.g., Fazio et al. 2004). In the latter case, 14 are removed with a minimum size criterion (set by the $3.6 \mu \mathrm{m}$ FWHM), and 12 others avoided with limits to the mid-IR colors: star-forming galaxies will have [3.6] - [8] $>3.35$ 
Table 1

Catalog of AGB-dominated Clusters ${ }^{\mathrm{a}}$

\begin{tabular}{|c|c|c|c|c|c|c|c|c|c|c|c|c|c|c|}
\hline ID & $\begin{array}{l}\text { R.A. } \\
\text { (deg) }\end{array}$ & $\begin{array}{l}\text { Decl. } \\
\text { (deg) }\end{array}$ & $\begin{array}{c}m_{B} \\
(\mathrm{mag})\end{array}$ & $\begin{array}{c}d m_{B} \\
(\mathrm{mag})\end{array}$ & $\begin{array}{c}m_{V} \\
(\mathrm{mag})\end{array}$ & $\begin{array}{c}d m_{V} \\
(\mathrm{mag})\end{array}$ & $\begin{array}{c}m_{R} \\
(\mathrm{mag})\end{array}$ & $\begin{array}{c}d m_{R} \\
(\mathrm{mag})\end{array}$ & $\begin{array}{c}m_{I} \\
(\mathrm{mag})\end{array}$ & $\begin{array}{c}d m_{I} \\
(\mathrm{mag})\end{array}$ & $\begin{array}{c}m_{J} \\
(\mathrm{mag})\end{array}$ & $\begin{array}{c}d m_{J} \\
(\mathrm{mag})\end{array}$ & $\begin{array}{c}m_{H} \\
(\mathrm{mag})\end{array}$ & $\begin{array}{c}d m_{H} \\
(\mathrm{mag})\end{array}$ \\
\hline 1 & 185.7518 & 15.7765 & 21.158 & 0.103 & 19.503 & 0.101 & 17.987 & 0.100 & 16.842 & 0.100 & 14.918 & 0.050 & 14.312 & 0.050 \\
\hline 2 & 185.709 & 15.7771 & 22.275 & 0.250 & 21.813 & 0.278 & 20.462 & 0.178 & 19.914 & 0.162 & 18.350 & 0.080 & 17.677 & 0.084 \\
\hline 3 & 185.727 & 15.7849 & 21.667 & 0.135 & 21.215 & 0.153 & 20.770 & 0.176 & 20.241 & 0.145 & 18.573 & 0.072 & 17.526 & 0.073 \\
\hline 4 & 185.741 & 15.7898 & 20.616 & 0.156 & 20.405 & 0.182 & 20.211 & 0.266 & 20.543 & 0.313 & 17.764 & 0.098 & 16.910 & 0.095 \\
\hline 5 & 185.674 & 15.7942 & 21.308 & 0.106 & 20.467 & 0.103 & 19.414 & 0.102 & 18.893 & 0.104 & 17.708 & 0.052 & 16.591 & 0.054 \\
\hline 6 & 185.771 & 15.8304 & 21.060 & 0.140 & 21.022 & 0.183 & 20.639 & 0.226 & 20.222 & 0.178 & 18.366 & 0.112 & 17.478 & 0.097 \\
\hline 7 & 185.674 & 15.8395 & 22.149 & 0.120 & 21.175 & 0.110 & 19.951 & 0.108 & 20.044 & 0.119 & 18.217 & 0.054 & 17.065 & 0.064 \\
\hline 8 & 185.679 & 15.846 & 19.739 & 0.100 & 18.393 & 0.100 & 17.151 & 0.100 & 16.603 & 0.100 & 15.093 & 0.050 & 14.512 & 0.050 \\
\hline 9 & 185.673 & 15.8458 & 20.974 & 0.103 & 20.036 & 0.102 & 19.213 & 0.102 & 19.477 & 0.112 & 18.165 & 0.055 & 16.818 & 0.056 \\
\hline 10 & 185.751 & 15.8538 & 21.258 & 0.270 & 20.197 & 0.167 & 19.199 & 0.140 & 18.725 & 0.115 & 16.672 & 0.060 & 15.922 & 0.060 \\
\hline 11 & 185.754 & 15.8549 & 20.137 & 0.190 & 19.544 & 0.174 & 18.787 & 0.158 & 18.884 & 0.158 & 17.061 & 0.074 & 16.339 & 0.074 \\
\hline 12 & 185.695 & 15.8575 & 20.330 & 0.106 & 19.520 & 0.106 & 18.797 & 0.106 & 18.923 & 0.108 & 17.497 & 0.061 & 16.837 & 0.062 \\
\hline 13 & 185.68 & 15.8627 & 20.242 & 0.101 & 18.773 & 0.100 & 17.740 & 0.100 & 17.500 & 0.101 & 16.041 & 0.050 & 15.226 & 0.050 \\
\hline 14 & 185.732 & 15.8659 & 19.969 & 0.158 & 19.262 & 0.138 & 18.163 & 0.117 & 17.887 & 0.109 & 16.074 & 0.053 & 15.316 & 0.053 \\
\hline 15 & 185.74 & 15.8689 & 20.281 & 0.107 & 18.773 & 0.101 & 17.738 & 0.101 & 17.481 & 0.100 & 16.060 & 0.050 & 15.262 & 0.050 \\
\hline 16 & 185.743 & 15.8714 & 21.781 & 0.111 & 20.994 & 0.111 & 19.964 & 0.108 & 20.352 & 0.126 & 18.411 & 0.057 & 17.180 & 0.063 \\
\hline 17 & 185.701 & 15.8738 & 22.956 & 0.151 & 21.840 & 0.126 & 21.192 & 0.138 & 20.157 & 0.105 & 19.253 & 0.069 & 17.479 & 0.058 \\
\hline ID & & & $\begin{array}{c}m_{K} \\
(\mathrm{mag})\end{array}$ & $\begin{array}{c}d m_{K} \\
(\mathrm{mag})\end{array}$ & $\begin{array}{c}m_{3.6} \\
(\mathrm{mag})\end{array}$ & $\begin{array}{l}d m_{3.6} \\
\text { (mag) }\end{array}$ & $\begin{array}{c}m_{4.5} \\
(\mathrm{mag})\end{array}$ & $\begin{array}{l}d m_{4.5} \\
(\mathrm{mag})\end{array}$ & $\begin{array}{c}m_{5.8} \\
(\mathrm{mag})\end{array}$ & $\begin{array}{l}d m_{5.8} \\
(\mathrm{mag})\end{array}$ & $\begin{array}{c}m_{8} \\
\text { (mag) }\end{array}$ & $\begin{array}{c}d m_{8} \\
\text { (mag) }\end{array}$ & $\begin{array}{c}m_{24} \\
(\mathrm{mag})\end{array}$ & $\begin{array}{l}d m_{24} \\
(\mathrm{mag})\end{array}$ \\
\hline 1 & & & 14.247 & 0.050 & 13.400 & 0.112 & 13.270 & 0.112 & 13.360 & 0.113 & 13.468 & 0.116 & 12.664 & 0.079 \\
\hline 2 & & & 16.748 & 0.068 & 15.408 & 0.116 & 15.080 & 0.115 & 14.063 & 0.122 & 12.987 & 0.125 & 10.762 & 0.072 \\
\hline 3 & & & 16.885 & 0.065 & 15.583 & 0.114 & 15.164 & 0.113 & 14.175 & 0.123 & 12.918 & 0.125 & 11.747 & 0.076 \\
\hline 4 & & & 16.643 & 0.100 & 15.465 & 0.130 & 15.448 & 0.132 & 14.332 & 0.160 & 12.676 & 0.157 & 11.212 & 0.081 \\
\hline 5 & & & 15.988 & 0.052 & 15.250 & 0.112 & 14.768 & 0.112 & 14.043 & 0.115 & 13.236 & 0.114 & 11.290 & 0.071 \\
\hline 6 & & & 16.500 & 0.071 & 16.538 & 0.149 & 15.979 & 0.126 & 15.064 & 0.161 & 14.310 & 0.257 & 12.894 & 0.109 \\
\hline 7 & & & 15.972 & 0.061 & 15.917 & 0.114 & 15.521 & 0.115 & 14.653 & 0.124 & 13.619 & 0.118 & 12.404 & 0.080 \\
\hline 8 & & & 14.212 & 0.050 & 13.656 & 0.112 & 13.555 & 0.112 & 13.447 & 0.113 & 13.684 & 0.119 & 12.945 & 0.087 \\
\hline 9 & & & 16.026 & 0.054 & 15.974 & 0.114 & 15.344 & 0.113 & 14.386 & 0.120 & 13.570 & 0.117 & 11.498 & 0.072 \\
\hline 10 & & & 15.352 & 0.057 & 14.503 & 0.114 & 14.190 & 0.113 & 13.361 & 0.121 & 12.066 & 0.131 & 10.632 & 0.073 \\
\hline 11 & & & 15.817 & 0.067 & 15.098 & 0.121 & 14.964 & 0.120 & 13.892 & 0.123 & 12.518 & 0.123 & 11.462 & 0.078 \\
\hline 12 & & & 16.166 & 0.057 & 15.655 & 0.118 & 15.242 & 0.115 & 15.231 & 0.139 & 13.294 & 0.116 & 11.251 & 0.072 \\
\hline 13 & & & 14.525 & 0.050 & 14.036 & 0.112 & 13.750 & 0.112 & 13.788 & 0.114 & 13.189 & 0.114 & 13.324 & 0.094 \\
\hline 14 & & & 14.741 & 0.052 & 13.985 & 0.113 & 13.755 & 0.113 & 13.091 & 0.119 & 11.470 & 0.121 & 10.988 & 0.078 \\
\hline 15 & & & 14.606 & 0.050 & 14.037 & 0.112 & 13.776 & 0.112 & 13.868 & 0.116 & 13.608 & 0.131 & 13.202 & 0.103 \\
\hline 16 & & & 16.331 & 0.068 & 15.858 & 0.114 & 15.546 & 0.115 & 14.649 & 0.122 & 13.973 & 0.135 & 11.836 & 0.075 \\
\hline 17 & & & 17.216 & 0.056 & 15.953 & 0.114 & 15.608 & 0.115 & 14.949 & 0.132 & 13.962 & 0.122 & 11.574 & 0.072 \\
\hline
\end{tabular}

Note. ${ }^{\text {a }}$ All photometric measurements are presented relative to Vega.

(tracing hot dust and PAH emission) and [8] - [24] 22.4 (see Boyer et al. 2011).

Contamination from background galaxies is otherwise naturally limited by the optically thick disk of M100 (Holwerda et al. 2005). As a secondary safeguard we use the few known background galaxies detected by Holwerda et al. (2005) between 70" and 140" in archival Hubble Space Telescope/WFPC2 imaging to construct a composite SED against which all candidates are compared. These galaxies are on average one magnitude dimmer at $3.6 \mu \mathrm{m}$ than clusters in our final sample and have

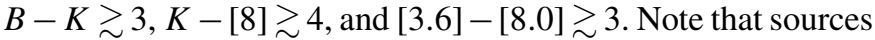
falling nearest the disk edge where the dust column density is lower show less strongly the suppression at optical wavelengths characteristic of foreground disk extinction. We therefore expect the above mid-IR color criteria to be a stronger limit for these.

According to the detection rate of active galactic nuclei behind the Large Magellanic Cloud (LMC) and Small Magellanic Cloud we expect quasar contamination to be minimal (less than 2 in the field studied here; Kozlowski \& Kochanek 2009), and few candidates approach the limit $R-K \sim 4$ used to detect obscured quasars with similar mid-IR colors (e.g., Lacy et al. 2004; Gilkman et al. 2007). Embedded star formation in young massive clusters and $\mathrm{HII}$ regions illuminating the surrounding dust are perhaps stronger potential contaminants. Our imposed criterion [3.6] - [8] $<3.35$ already avoids many such objects, four of which also have detectable $\mathrm{H} \alpha$ emission. To select against any more deeply embedded mid-IR bright clusters we impose a $3 \sigma$ threshold in the optical $B V R I$ bands. With 27 such sources removed, the remaining 17 visually confirmed clusters (Table 1) exhibit [3.6] - [8] colors consistent with emission from circumstellar material (see Figure 2), as expected around AGB stars undergoing high mass loss (e.g., Groenewegen et al. 2009; Sargent et al. 2011; Srinivasan et al. 2011).

\subsection{Sample Description}

By construction, selected clusters originate in zones with little to no emission from PAH and hot dust at 3.6 and $4.5 \mu \mathrm{m}$ and so they should also be largely removed of locations in the disk susceptible to the effects of reddening and extinction by diffuse dust (see Figure 1). Extracluster extinction is therefore expected to be minimal, especially since the final 17 clusters 

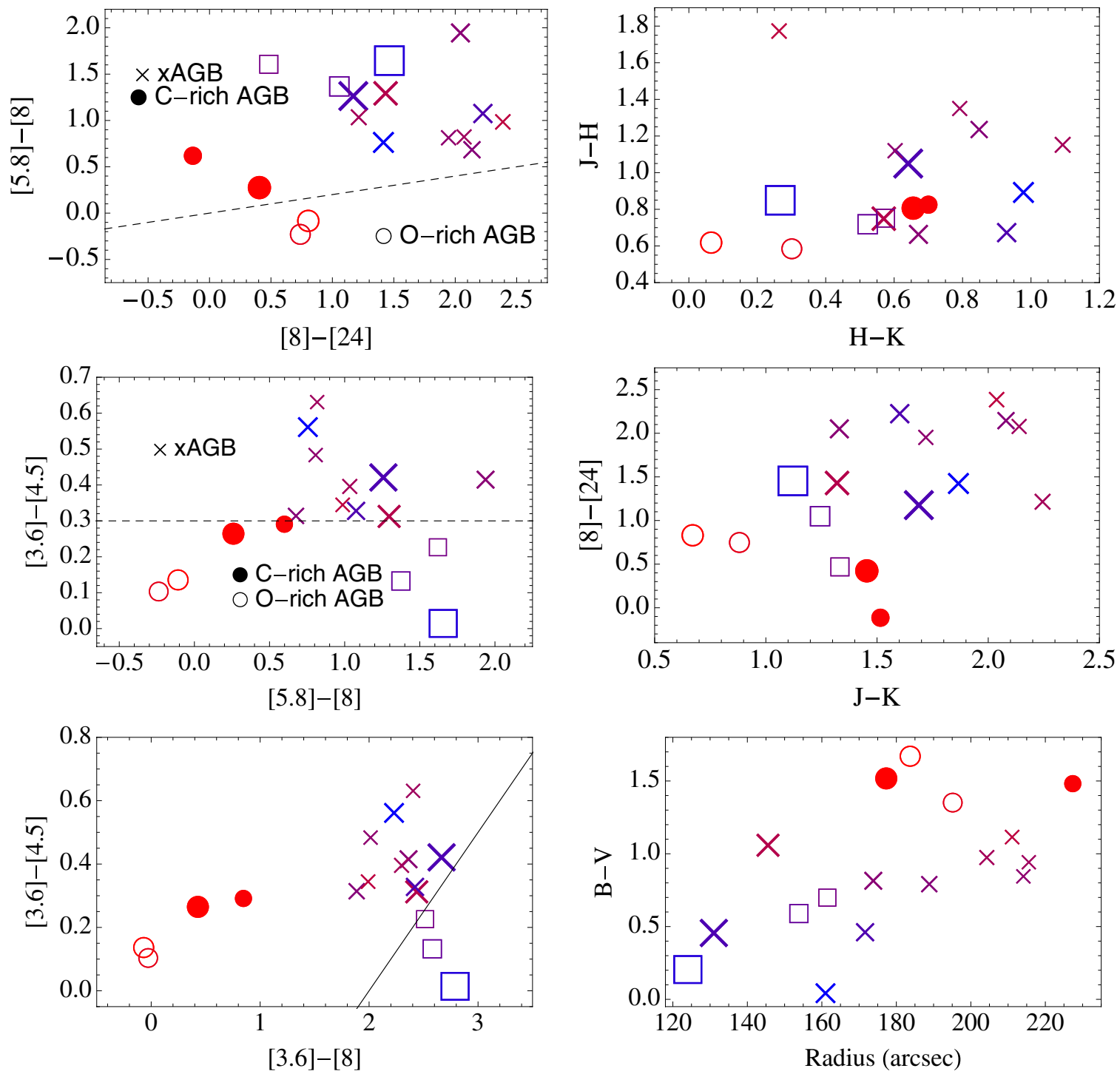

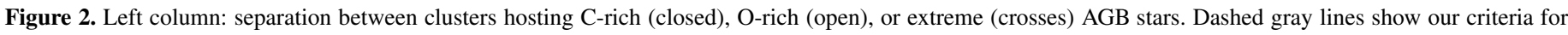

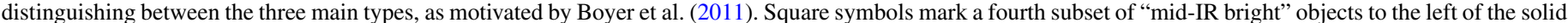

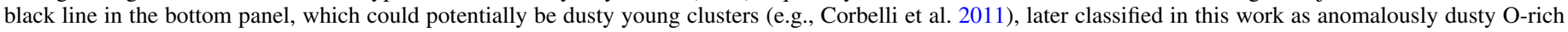

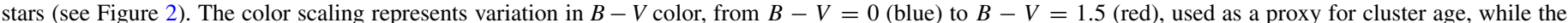

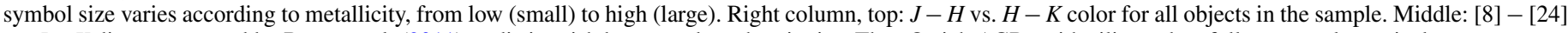

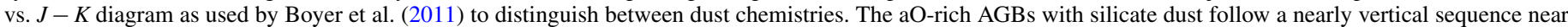

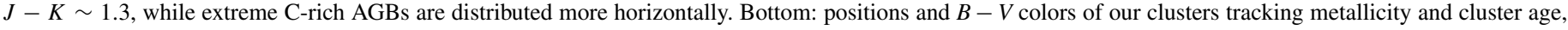
respectively.

(A color version of this figure is available in the online journal.)

fall in the outer disk where the dust column density is low. As revealed in Section 4.1, our mid-IR measurements are consistent with emission from the circumstellar dust shell produced, and illuminated, by the AGB star itself, rather than from a diffuse dust component emitting reprocessed radiation from cluster stars. We take the measured red $B-V$ colors (Figure 2) as characteristic of intrinsically red objects with a spread in ages between 0.2 and 1 Gyr (Bruzual 2007; Mouhcine \& Lançon 2002).

Our sample is evidently composed of coeval massive cluster complexes with $M>10^{6} M_{\odot}$ (e.g., Trancho et al. 2007; referred to simply as clusters in what follows), given the unavoidable bias toward the brightest sources at this distance and our requirement for optical detections. The depth of the potential of the cluster complex relative to the shallow outer disk provides a natural way to avoid dispersal at these late ages (i.e., Fellhauer \& Kroupa
2005), while interaction with the companion galaxy suggests a plausible seed for the formation of such massive complexes (e.g., Larsen 2004).

\section{VARIATION IN THE AGB CONTRIBUTION}

\subsection{Distinction between $O$-rich, $C$-rich, and Extreme AGB Stars}

The varying degrees of circumstellar dust extinction expected around AGBs are potentially closely related to the chemistry of the star and the mass of its progenitor. C-rich stars will be more highly extincted than O-rich stars for the same mass-loss rate, given the higher opacities of the carbonaceous grains produced in their atmospheres (e.g., Suh 2000; Pégourié 1988; Mouhcine 2002). The highest levels of extinction occur in the extreme AGB phase, during which the star undergoes its highest mass- 
loss rates. Following Boyer et al. (2011), we use mid-IR colors to differentiate between clusters dominated by C-rich, O-rich, or extreme AGB stars (Figure 2).

Although age and metallicity variations between clusters may introduce scatter relative to the separation in, e.g., the LMC (Boyer et al. 2011), the different AGB star types cleanly separate by their mid-IR colors. Note that, without spectral confirmation, these particular classifications are subject to interpretation. Following Kastner et al. (2008) and Groenewegen et al. (2009), for example, the extreme AGBs here would be classified as C-rich (and see Ferraro et al. 1995).

Clusters also show good correspondence with the NIR colors of isolated AGBs (top right panels of Figure 2), despite the higher contribution from the rest of the cluster members than in the mid-IR. These very red NIR colors supply additional confirmation that our clusters are dominated by AGBs undergoing high mass-loss rates (e.g., Gonzalez-Lopezlira 2010); even with the implied color excess $E(B-V)$ for ages between 1 and $10 \mathrm{Myr}$, we find that the NIR colors remain consistent with $0.2-1.2$ Gyr old clusters.

Our classifications are confirmed in the [8] - [24] versus $J-K$ diagram (middle; cf. Boyer et al. 2011). Based on this diagram our "mid-IR bright" objects can be associated with the anomalously dusty O-rich (aO-rich) AGBs identified by Boyer et al. (2011). This is also favored by our combined optical and mid-IR view: where the red [3.6]-[8] colors of dust-reprocessed emission from young embedded clusters would coincide with dust-reddened $B-V$ colors, these objects have some of the bluest optical colors in the sample. These objects may therefore be the O-rich counterpart to the extreme C-rich AGBs in our sample.

The bottom right panel of Figure 2 depicts the dependence of AGB type on mass and metallicity, in line with the mechanisms that influence the $\mathrm{C} / \mathrm{O}$ ratio (see, e.g., van Loon et al. 2005). The dustiest AGBs notably sit in younger clusters, where AGB progenitors are of higher mass than at older ages. This accounts for the enhanced dust production in these stars, if the implied longer pulsation period for these high masses leads to higher mass-loss rates. In contrast, the less dusty C-rich and O-rich appear less often here and only at the outer radii as a result of our mid-IR and optical detection thresholds and large aperture.

\subsection{Extinction in the $K$ Band}

The impact of the different AGB star dust chemistries and mass-loss rates is clear in Figure 3 where clusters form a sequence that becomes progressively redder in $K-$ [8] color while becoming bluer in $B-K$ color. The optical-NIR color traces the fractional flux contribution of AGB stars relative to the other (less evolved) cluster members, which dominate in the optical. The $K-[8]$ color meanwhile serves to measure the amount of dust produced around the AGB stars, in the sense that enhanced $8 \mu \mathrm{m}$ dust emission and reduced $K$-band brightness due to extinction both drive reddening in $K-$ [8].

To reproduce the downward trend in this color-color diagram that separates the C-rich from the O-rich AGBs and the dustiest AGBs from those that are less dusty requires a decrease in the $K$-band cluster light from the top left to the bottom right (depicted by the overlaid dashed lines of $K$-band dimming at arbitrary fixed $B$ and [8] brightness). This can be most easily explained by an enhanced obscuration of the AGB stars as the optical depth of their dust shells increases.

The stellar photosphere or spectral type of the AGB star also plays a role in determining the location within this diagram

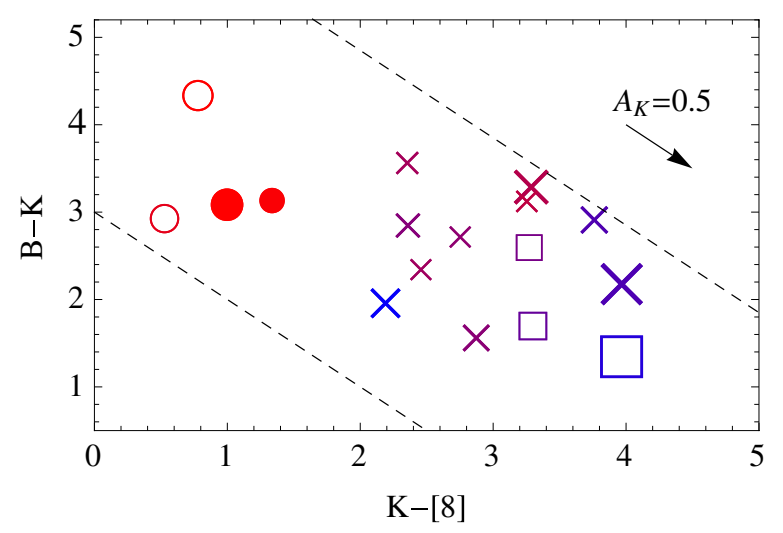

Figure 3. $B-K$ vs. $K-[8]$ color for the final cluster sample. Dashed lines illustrate decreasing $K$-band brightness at fixed $B$ and $8 \mu \mathrm{m}$ magnitudes. Symbol types, sizes, and colors are as in Figure 2.

(A color version of this figure is available in the online journal.)

(e.g., Sargent et al. 2011). This is contained in our distinction between $\mathrm{C}$-rich and O-rich chemistries and so, in this sense, it is largely indistinguishable from the implied dust extinction. As a result of the different properties of the dust grains produced in the atmospheres of C-rich and O-rich stars, the former will be relatively more obscured for the same mass-loss rate.

Other interpretations for the downward trend in Figure 3 are either less viable or precluded by construction. By avoiding sources with mid-IR emission from dust heated by young stars we have removed the potential that the bluer $B-K$ colors arise at an age before the onset of AGBs, as well as the possibility that reddest $K-$ [8] colors trace extracluster dust heated to higher temperatures.

In addition, the decrease in $B-K$ is inconsistent with fluctuations to lower AGB star number densities (resulting in lower NIR fluxes relative to the optical), as the corners of the diagram would also be filled, since $B-K$ would be independent of $K-8$ in this case.

This trend is also not driven by intra-cluster dust from past mass loss by earlier AGBs at younger cluster ages, which would have reversed the observed red-to-blue progression in $B-V$ colors along the downward sequence of increasing extinction. Instead, clusters that are blue in $B-K$ are also blue in $B-V$. As noted in Section 4.1, this reflects a genuine dependence on cluster age through the mass of the progenitor; at young cluster ages AGBs will evolve from higher mass stars resulting in AGBs with higher mass-loss rates and hence heavier extinction.

\subsection{Implications for Age and Mass Estimation}

The trend in Figure 3 implies that the contribution of dusty AGBs in the $K$ band is much lower than expected from the $B$-band cluster emission due to circumstellar extinction. Since optical-NIR colors like $B-K$ are commonly used as age indicators for intermediate-age populations (Bruzual 2007; Mouhcine 2002), this suggests that intrinsic extinction can significantly affect the predicted ages of AGB-dominated populations, as explored by Mouhcine (2002). Age estimation and classification from NIR colors should be likewise affected (e.g., Nowotny et al. 2011). More consequentially, models with relatively "bare" AGBs, less extincted by circumstellar dust, will underpredict the stellar $M / L$ for intermediate-age populations. This is demonstrated in Figure 4, showing composite SEDs for the four sets of clusters studied here, those that host O-rich and C-rich AGBs and those with relatively more obscured extreme C-rich and 


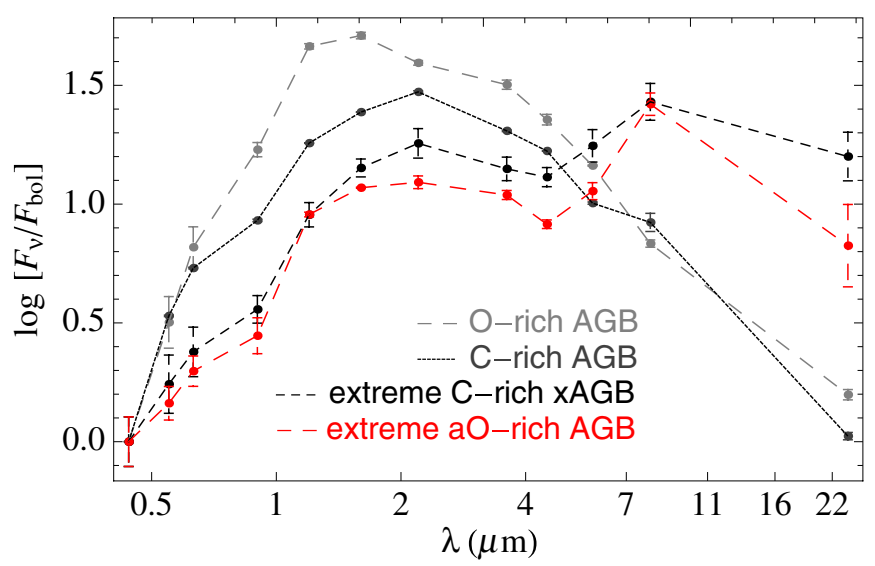

Figure 4. Composite SEDs normalized at $0.4 \mu \mathrm{m}$. For a given type, we take the average of SEDs each normalized to its bolometric flux $F_{\text {bol }}$. Error bars represent the dispersion in the measurements for each type.

(A color version of this figure is available in the online journal.)

aO-rich AGBs. In the latter two cases, circumstellar dust clearly shifts the cluster emission from short to long wavelengths so that the NIR contribution of AGBs appears significantly lower than in the case of relatively "bare" O-rich AGBs. The C-rich AGBs also show a depression in the NIR relative to the O-rich case. This amounts to $0.12 \mathrm{dex} \approx 0.3 \mathrm{mag}$ extinction in the $K$-band for C-rich stars compared to O-rich stars and on average $\sim 0.5$ dex extinction for the dustiest AGBs (or as much as $1 \mathrm{mag}$, also judging by the offset in $B-K$ color in Figure 3 ).

The difference in SED shapes with and without strong AGB star extinction can explain the apparent compatibility found by Kriek et al. (2010) between the SEDs of post-starburst galaxies and the older generation of Bruzual \& Charlot (2003) models, where AGBs contribute $\sim 30 \%-40 \%$ in $\mathrm{K}$ at $1 \mathrm{Gyr}$ rather than $\sim 70 \%$ in the recent, updated version of these models. But the agreement found by Kriek et al. (2010) would seem to be largely coincidental: taking the extinction measured from the difference in $K$-band light in the undimmed and dimmed cases, then a $70 \%$ contribution from AGB stars in the $K$ band $(30 \%$ from the cluster) will be reduced to $\sim 35 \%$ as the result of circumstellar dust extinction.

\section{CONCLUDING REMARKS}

We have presented evidence that the fractional contribution of AGBs to intermediate-age populations, a contentious prediction of SPS models, is significantly affected by the presence of the dusty envelope produced during this stage. The extinction generated by dust shells around AGBs in $\sim 1$ Gyr old clusters (on average $0.5 \mathrm{mag}$ ) is sensitive to the type of AGB star: C-rich AGBs produce shells with higher optical depths than their Orich counterparts and the high mass-loss rates of extreme AGBs lead to even higher obscuration. Consequently, population ages estimated from optical-NIR colors may be underestimated (as predicted by Mouhcine 2002), leading also to underprediction of the stellar $M / L$. The range in circumstellar extinction revealed here may account for the fact that SPS models with very different AGB star contributions each seem to fit a diversity of observations well (cf. Maraston et al. 2006; van der Wel et al. 2006; Kriek et al. 2010).

S.E.M. thanks Arjen van der Wel for discussion and Angela Adamo and Anibal Garcia for their helpful comments on the manuscript. E.A. and A.B. thank the Centre National d'Etudes Spatiales for financial support.

\section{REFERENCES}

Boyer, M. L., Srinivasan, S., van Loon, J., et al. 2011, AJ, 142, 103 Bruzual, G. 2007, in IAU Symp. 241, Stellar Populations as Building Blocks of Galaxies, ed. A. Vazdekis \& R. Peletier (Cambridge: Cambridge Univ. Press), 125

Bruzual, G., \& Charlot, S. 2003, MNRAS, 344, 1000

Calzetti, D., Kennicutt, R. C., Engelbracht, C. W., et al. 2007, ApJ, 666, 870

Corbelli, E., Giovanardi, C., Palla, F., \& Verley, S. 2011, A\&A, 528, 116

Dale, D. A., Bendo, G. J., Engelbracht, C. W., et al. 2005, ApJ, 633, 857

Dale, D. A., Gil de Paz, A., Gordon, K. D., et al. 2007, ApJ, 655, 863

Fazio, G. G., Ashby, M. L. N., Barmby, P., et al. 2004, ApJS, 154, 39

Fellhauer, M., \& Kroupa, P. 2005, ApJ, 630, 879

Ferraro, F. R., Fusi Pecci, F., Testa, V., et al. 1995, MNRAS, 272, 391

Freedman, W. L., Madore, B. F., Gibson, B. K., et al. 2001, ApJ, 553, 47

Gilkman, E., Helfand, D. J., White, R. L., et al. 2007, ApJ, 667, 673

Girardi, L., \& Marigo, P. 2007, A\&A, 462, 237

Gonzalez-Lopezlira, R. A., Bruzual, G., Charlot, S., Ballesteros-Paredes, J., \& Loinard, L. 2010, MNRAS, 403, 1213

Groenewegen, M. A. T. 2006, A\&A, 448, 181

Groenewegen, M. A. T., Sloan, G. C., Soszynski, I., \& Petersen, E. A. 2009, A\&A, 506, 1277

Grosbol, P., \& Dottori, H. 2011, in Stellar Clusters \& Associations: A RIA Workshop on Gaia, ed. E. J. Alfaro Navarro, A. T. Gallego Calvente, \& M R. Zapatero Osorio, 123 (http://sca.iaa.es/content/proceedings)

Holwerda, B. W., Gonzalez, R. A., Allen, R. J., \& van der Kruit, P. C. 2005, AJ, 129,1396

Jackson, D. C., Skillman, E. D., Gehrz, R. D., Polomski, E., \& Woodward, C. E. 2007, ApJ, 656, 818

Kastner, J. H., Thorndike, S. L., Romanczyk, P. A., et al. 2008, AJ, 136, 1221

Kennicutt, R. C., Jr., Armus, L., Bendo, G., et al. 2003, PASP, 115, 928

Kozlowski, S., \& Kochanek, C. S. 2009, ApJ, 701, 508

Kriek, M., Labbé, I., Conroy, C., et al. 2010, ApJ, 722, L64

Lacy, M., Storrie-Lombardi, L. J., Sajina, A., et al. 2004, ApJS, 154, 166

Larsen, S. S. 2004, A\&A, 416, 537

Maraston, C. 2005, MNRAS, 362, 799

Maraston, C., Daddi, E., Renzini, A., et al. 2006, ApJ, 652, 85

Marigo, P., Girardi, L., Bressan, A., et al. 2008, A\&A, 482, 883

Matsuura, M., Barlow, M. J., Zijlstra, A. A., et al. 2009, MNRAS, 396, 918

Meidt, S. E., Schinnerer, E., Knapen, J. H., et al. 2012, ApJ, 744, 17

Mouhcine, M. 2002, A\&A, 394, 125

Mouhcine, M., \& Lançon, A. 2002, A\&A, 393, 149

Moustakas, J., Kennicutt, R. C., Tremonti, C. A., et al. 2010, ApJS, 190, 233

Nowotny, W., Aringer, B., Hoefner, S., \& Lederer, M. T. 2011, A\&A, 529, 129

Pégourié, B. 1988, A\&A, 194, 335

Reach, W. T., Megeath, S. T., Cohen, M., et al. 2005, PASP, 117, 978

Sanders, D. B., Salvato, M., Aussel, H., et al. 2007, ApJS, 172, 86S

Sargent, B. A., Srinivasan, S., \& Meixner, M. 2011, ApJ, 728, 93

Sargent, B. A., Srinivasan, S., Meixner, M., et al. 2010, ApJ, 716, 878

Sheth, K., et al. 2010, PASP, 122, 1397

Srinivasan, S., Meixner, M., Leitherer, C., et al. 2009, AJ, 137, 4810

Srinivasan, S., Sargent, B., \& Meixner, M. 2011, A\&A, 532, A54

Suh, K.-W. 2000, MNRAS, 315, 740

Trancho, G., Bastian, N., Miller, B. W., \& Schweizer, F. 2007, ApJ, 664, 284

van der Wel, A., Franx, M., Wuyts, S., et al. 2006, ApJ, 652, 97

van Loon, J. T., Marshall, J. R., \& Zijlstra, A. A. 2005, A\&A, 442, 597 